\title{
Absence of attentional capture in parallel search is possible: A failure to replicate attentional capture in a non-singleton target search task
}

\author{
Carolin Wienrich • Markus Janczyk
}

Published online: 30 July 2011

(C) Psychonomic Society, Inc. 2011

\begin{abstract}
The notion of a singleton versus a feature search mode (Bacon \& Egeth, 1994) in visual search is generally widely accepted. Yet Theeuwes (2004) claimed a different, potentially more parsimonious position. He suggested that the size of an attentional window is under top-down control and that salient distractors within this window capture attention. We report on four experiments. The first two experiments represent our various initial attempts to replicate the crucial Experiment 1 in the Theeuwes (2004) study, all of which were not successful. In Experiment 3, we showed that our distractor was able to yield attentional capture in a situation promoting the use of the singleton search mode (Bacon \& Egeth, 1994). Experiment 4 was similar to Experiment 1 but incorporated further details that may account for the discrepant findings. Still, no attentional capture was found. In sum, it was not possible to replicate a crucial piece of evidence for the attentional window hypothesis, and our results are more consistent with the assumption of two different search modes.
\end{abstract}

Keywords Attentional capture - Visual search · Top-down . Bottom-up $\cdot$ Salience $\cdot$ Singleton

C. Wienrich $(\bowtie)$

Department of Psychology and Ergonomics,

Berlin Institute of Technology,

Franklinstraße 28/29,

10587 Berlin, Germany

e-mail: carolin.wienrich@tu-berlin.de

M. Janczyk

Department of Psychology III,

University of Würzburg,

Röntgenring 11,

97070 Würzburg, Germany
The ability to attend to specific objects in the environment while ignoring other (irrelevant) objects is referred to as selective attention. Still, there are instances when irrelevant objects capture our attention. In the visual domain, this phenomenon of attentional capture is widely investigated, and we elaborate on this in the next section. We then introduce two accounts for when and why such attentional capture actually occurs.

\section{Attentional capture}

The existence of visual attentional capture has been convincingly shown in a series of studies using the additional singleton paradigm (e.g., Theeuwes, 1991, 1992, 1994). In this paradigm, participants may search, for example, for a green circle surrounded by red circles (i.e., the green circle is a color singleton) in the distractor absent condition. In contrast, in the distractor present condition, one of the red circles is replaced by a red square - the distractor (which is a form singleton). Attentional capture is manifested in the finding that response times (RTs) are longer in the distractor present than in the distractor absent condition. According to the bottom-up (or stimulus-driven) view, attentional capture depends only on the salience of the stimuli: Attention is first directed to the most salient item in the search display and must then be disengaged from the erroneously attended distractor location. This disengagement is reflected in the longer RTs when a distractor is present (Theeuwes, 1991, 1992, 1994). Other researchers have, instead, advocated a crucial role for top-down (or goaldriven) influence (e.g., Bacon \& Egeth, 1994; Folk, Remington, \& Johnston, 1992). We will discuss one prominent theory of the latter class in greater detail in the next section. 


\section{Two different search modes ...}

Bacon and Egeth (1994), following Pashler (1988), reinterpreted the basic findings of Theeuwes (1991, 1992, 1994). In particular, they suggested the existence of two different search modes. In the singleton search mode, the observer "identifies elements that differ from their backgrounds" (Bacon \& Egeth, 1994, p. 486). This mode is applied if the target is unique against other items in the search display in one dimension (i.e., a singleton), but as a consequence, the presence of an additional singleton interferes with search performance. They suggested that although participants in the Theeuwes (1992) study knew the exact identity of the target, they nevertheless used the singleton search mode, which made them prone to interference from the additional singleton. If, however, the target is not a singleton in its dimension, participants are forced to use what Bacon and Egeth termed the feature search mode. Here, attention is set to a specific feature, and only stimuli that share such a feature can capture attention involuntarily. In fact, when they had their participants search for a specific shape that was not a singleton (their Experiments 2 and 3) no attentional capture by a color singleton was observed. Such findings were later replicated and extended by Lamy and Egeth (2003), although it appears that non-static distractors (such as abrupt onsets) can capture attention even in the feature search mode. However, according to this theory, top-down settings can override stimulus-driven attentional capture.

\section{... or the size of an attentional window?}

Theeuwes (2004) suggested a surprisingly parsimonious alternative to the search mode approach of Bacon and Egeth (1994): In his view, it is not a specific search mode that is top-down selected, but only the size of an attentional window that is adjusted. With easy search tasks, an observer may adopt a large attentional window yielding parallel search. The more difficult the search task, the more likely it is that an observer will reduce the size of this window, thereby producing a serial search. Furthermore and irrespective of the window's size, it is assumed that attention is initially and unavoidably directed to the most salient item within the attentional window. Hence, only distractors inside this window can interfere with target search, and as a consequence, parallel search (driven by a wide attentional window) is always susceptible to attentional capture, while serial search is not. In support of this view, Theeuwes (2004) reported two experiments. In his Experiment 1, he increased the salience of the target (and the distractor) by increasing the set size (i.e., displaying either 12 or 20 items on each trial; in the Bacon and Egeth (1994) study, set size was varied from 5 to 9). This should increase the homogeneity of the search display, making the search easier, and thereby encourage a wide attentional window. Indeed, the absence of a set size effect suggested parallel search, and, as was predicted, attentional capture from a color singleton occurred. Notably, this was the case even though the target was not a (form) singleton, what should encourage the use of the feature search mode (Bacon \& Egeth, 1994). As a consequence, such a finding is difficult to explain within the search mode approach. In Experiment 2, Theeuwes (2004) made the search task more difficult by decreasing the target's salience through using set sizes of 5 and 9 to encourage a small attentional window. Here, the set size effect suggested a (partially) serial search, but the distractor effect was no longer reliable. It should be noted that additional studies exist in which the size of the attentional window was manipulated explicitly (Belopolsky \& Theeuwes, 2010; Belopolsky, Zwaan, Theeuwes, \& Kramer, 2007). For example, in Belopolsky and Theeuwes, the search task was to be started only upon a go-cue, the identification of which required either a wide (diffuse condition) or a small (focused condition) attentional window. In these studies, more signs of attentional capture were found in the diffuse condition, where participants were assumed to have established a wide attentional window. These results were interpreted as support for the attentional window hypothesis.

Leber and Egeth (2006) emphasized that according to the attentional window hypothesis, it should not be possible to observe parallel search (indicating a wide attentional window), without interference from an irrelevant and sufficiently salient distractor. In their experiment, two groups of participants worked, in a training phase, on visual search tasks that allowed application of either the singleton or the feature search mode (Bacon \& Egeth, 1994). In a subsequent test phase, all participants worked on a search task, where the target was a form singleton, thus allowing the application of the singleton search mode. Crucially, despite parallel search, the group that had previously been trained on the feature search mode did not show any signs of attentional capture from an irrelevant color singleton. As such, the results of Leber and Egeth violate one of the crucial assumptions of the attentional window hypothesis.

\section{The present experiments}

The attentional window hypothesis proposed by Theeuwes (2004) provides a parsimonious and interesting specification for the circumstances of attentional capture to occur. In the research presented here, we attempted to replicate Theeuwes's (2004) Experiment 1 but were unable to do so despite multiple attempts and experimental variations. To anticipate, we always observed a parallel search pattern, but we never found an interfering distractor effect (Experiments 1, 
2, and 4); a distractor effect was present only when the target was a form singleton (Experiment 3), thus possibly allowing the use of the singleton search mode (Bacon \& Egeth, 1994).

\section{General method}

The basic experimental setup and data analyses were the same for all the experiments. Deviations from these procedures are mentioned in the respective Method sections when necessary.

\section{Participants}

In most experiments, 8 (12 in Experiment 4) students from Martin Luther University Halle-Wittenberg, University of Würzburg, or Dortmund University of Technology participated. The only exception was Experiment 1d, where 10 high school students from Dortmund participated. Participants either volunteered or were paid course credit in exchange. All participants reported normal or corrected-tonormal vision, and no one participated in more than one experiment. Except for a few participants from Experiment $1 \mathrm{a}$, no one had prior experience with search tasks such as those employed here.

\section{Apparatus}

Stimulus presentation and response recording were controlled with a standard PC (19-in. monitor at Halle; 17-in. monitor at Würzburg and Dortmund). A chinrest was used throughout all experiments to maintain a constant viewing distance $(45 \mathrm{~cm})$.

\section{Stimuli}

In each experimental condition, either 12 or 20 (= set size) geometrical shapes appeared equally spaced on the outline of either one or two concentric imaginary circles centered at fixation (radius $5.9^{\circ}$ for set size 12 ; radii $5.9^{\circ}$ and $3.7^{\circ}$ for set size 20$)$ in a random order. Items were one square $\left(1.4^{\circ}\right.$ each side), one triangle $\left(2.3^{\circ}\right.$ each side), one diamond as the target $\left(45^{\circ}\right.$ rotated square; $1.4^{\circ}$ each side), and circles $\left(1.4^{\circ}\right.$ diameter). Each shape contained a small, slightly rotated $\left(22.5^{\circ}\right.$ from horizontal or vertical) line segment of $0.6^{\circ}$ length; only the line segment within the target diamond was perfectly horizontally or vertically aligned (see Fig. 1 for examples). Two different conditions were employed. In the distractor absent condition, all stimuli (including the line segments) were green $\left(56.5 \mathrm{~cd} / \mathrm{m}^{2}\right)$. In the distractor present condition, one circle was red $\left(19.0 \mathrm{~cd} / \mathrm{m}^{2}\right)$ - thus, a color singleton (the line segment inside was still green). The stimuli were presented against a black $\left(0.0 \mathrm{~cd} / \mathrm{m}^{2}\right)$ background.

\section{Procedure}

Participants were tested individually in experimental rooms at the Departments of Psychology at Martin Luther University Halle-Wittenberg, University of Würzburg, and Dortmund University of Technology. The participants' task was to report the orientation of the small line segment placed in the diamond by pressing the left or right CTRL key on the computer keyboard in response to a horizontal or vertical orientation, respectively. Each trial began by presenting a fixation cross for $550 \mathrm{~ms}$, followed by the search display. The display was cleared after the response or after $2,000 \mathrm{~ms}$ without a response. In the latter case, the trial was marked as incorrect (and error feedback was given for $1,000 \mathrm{~ms}$ during practice blocks). The next trial started after $1,000 \mathrm{~ms}$. Written instructions were given on the computer screen, emphasizing speed and accuracy. Each participant performed 256 trials, 128 with the red distractor circle and 128 without (distractor present vs. distractor absent). Half of the participants began with the distractor present condition, the other half with the distractor absent condition. Between the two blocks, participants were obliged to rest for at least $30 \mathrm{~s}$. The first 64 of the 128 trials per condition were unanalyzed practice trials, in which participants were given feedback on incorrect responses. Each session took about 20-30 min.

\section{Data analyses}

RT analyses were performed on correct trials only. In addition, outliers were excluded according to one of two rules. (1) RTs less than $200 \mathrm{~ms}$ and those exceeding the individual mean by more than 2.5 standard deviations in the respective design cell were regarded as outliers. (2) Only RTs longer than $1,700 \mathrm{~ms}$ were regarded as outliers (Theeuwes, 2004). Both rules yielded qualitatively the same results; thus, the reported analyses are based on the first rule. Mean correct RTs and error percentages were submitted to a $2 \times 2$ analysis of variance (ANOVA) with repeated measures on set size (12 vs. 20) and distractor (present vs. absent). Significance level was set to $\alpha=.05$ for all analyses, and sample effect sizes are given as $\eta_{\mathrm{p}}{ }^{2}$.

\section{Experiments 1a-1d}

Experiments 1a-1d were essentially four attempts to replicate Experiment 1 of Theeuwes (2004) with four independent samples of college or high school students from Halle and Dortmund. Thus, these experiments resemble Theeuwes's (2004) Experiment 1 in as much details as possible, based on what was mentioned in the Method section in Theeuwes (2004). 
Fig. 1 Examples of the stimulus displays used in Experiments 1 and 2: a set size 20 and $\mathbf{b}$ set size 12. All stimuli were green in the distractor absent condition; in the distractor present conditions, one circle was red ( = color singleton) In Experiment 3, the diamond was the only noncircle form ( $=$ form singleton); in Experiment 4, the line segments within the objects were white. The background was black (Experiments 1, 2 and 3) or dark gray (Experiment 4)
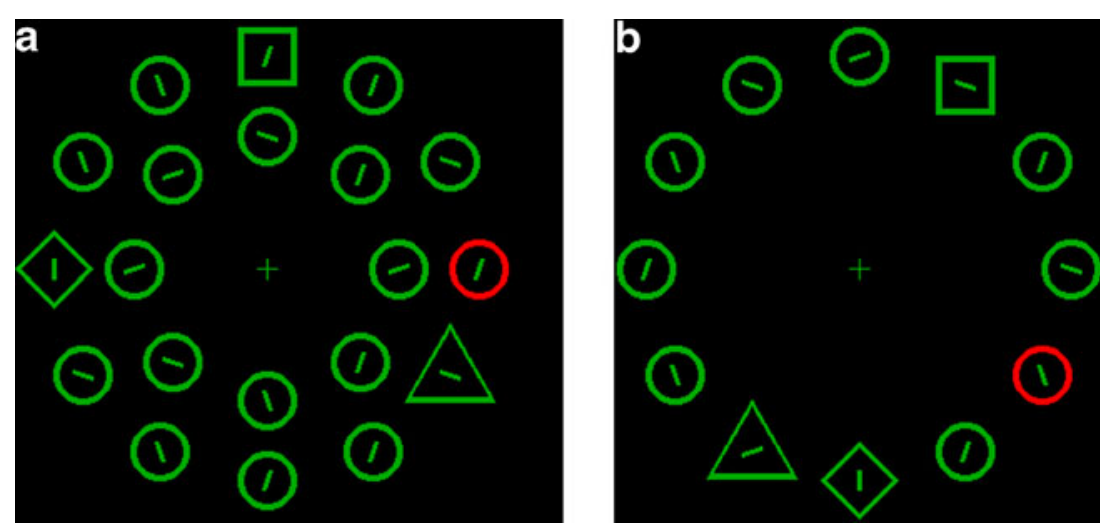

\section{Method}

Methodological and procedural details were as explicated in the General method section above.

Results and discussion

Mean correct RTs and mean error percentages for Experiments 1a-1d are summarized in Table 1, with the detailed ANOVA statistics given in Table 2. Across all experiments, no RT effect reached significance. While the absence of a set size effect was expected, the simultaneous absence of a distractor effect was surprising. An almost similar pattern arose from error data, where only in Experiment $1 \mathrm{~b}$ was the expected distractor effect found. This impression did not change after collapsing the data from all four experiments $(n=34$; for RT results, see Fig. 2, left panel). With experiment (1a-1d) as an additional between-subjects factor, the corresponding ANOVA did not yield significant effects for average mean correct, main effect of distractor: $F(1,30)=0.15, p=.70, \eta_{\mathrm{p}}{ }^{2}<.01$. The same held for mean error percentages, main effect of distractor: $F(1,30)=1.69, p=.20, \eta_{\mathrm{p}}{ }^{2}=.05$. Only the set size $\times$ distractor interaction (for mean error percentages) reached significance, $F(1,30)=5.23, p=.03, \eta_{\mathrm{p}}^{2}=.15$, suggesting a slightly larger distractor effect for a set size of 12 than of 20 .

The absence of a set size effect points to the expected parallel search as in Theeuwes (2004). In contrast, the distractor effect was not replicated for RTs and only once for error percentages. The absence of the distractor effect is difficult to explain within the attentional window framework (Theeuwes, 2004). Instead, it seems that it is indeed possible to obtain no distractor effect in parallel search (in agreement with Leber \& Egeth, 2006). There are two other noteworthy aspects of our data. First, the significant interac-
Table 1 Mean correct response times (RT) in milliseconds and mean error percentages in Experiments 1, 2, 3, and 4 as a function of set size and distractor. The data described as "Experiment 1 (fast)" included only the 8 fastest participants in Experiment 1

\begin{tabular}{|c|c|c|c|c|c|}
\hline \multirow[t]{2}{*}{ Experiment } & \multirow{3}{*}{$\begin{array}{l}\text { Distractor } \\
\text { absent }\end{array}$} & \multicolumn{2}{|c|}{ Mean Correct RT } & \multicolumn{2}{|c|}{ Mean Error Percentage } \\
\hline & & Set Size 12 & Set Size 20 & Set Size 12 & Set Size 20 \\
\hline \multirow[t]{2}{*}{ 1a } & & 1,164 & 1,159 & 4.7 & 6.6 \\
\hline & present & 1,143 & 1,133 & 8.2 & 5.1 \\
\hline \multirow[t]{2}{*}{$1 b$} & absent & 1,092 & 1,091 & 3.5 & 4.7 \\
\hline & present & 1,093 & 1,083 & 7.4 & 5.9 \\
\hline \multirow[t]{2}{*}{$1 \mathrm{c}$} & absent & 1,122 & 1,116 & 7.0 & 7.4 \\
\hline & present & 1,139 & 1,125 & 6.6 & 5.1 \\
\hline \multirow[t]{2}{*}{$1 d$} & absent & 1,119 & 1,115 & 5.0 & 7.5 \\
\hline & present & 1,106 & 1,093 & 14.1 & 13.1 \\
\hline \multirow[t]{2}{*}{1 (fast) } & absent & 963 & 967 & 3.1 & 4.7 \\
\hline & present & 992 & 979 & 5.5 & 5.1 \\
\hline \multirow[t]{2}{*}{$2 \mathrm{a}$} & absent & 1,105 & 1,139 & 9.0 & 6.6 \\
\hline & present & 1,138 & 1,110 & 8.6 & 9.0 \\
\hline \multirow[t]{2}{*}{$2 b$} & absent & 1,116 & 1,045 & 7.0 & 5.9 \\
\hline & present & 1,053 & 1,085 & 8.2 & 8.2 \\
\hline \multirow[t]{2}{*}{3} & absent & 605 & 606 & 3.1 & 2.3 \\
\hline & present & 640 & 648 & 0.8 & 4.3 \\
\hline \multirow[t]{2}{*}{4} & absent & 1,030 & 1,059 & 5.2 & 4.2 \\
\hline & present & 1,050 & 1,083 & 6.8 & 5.5 \\
\hline
\end{tabular}


Table 2 Detailed ANOVA statistics for Experiments 1, 2, 3, and 4 with mean correct response times (RTs) and mean error percentages as dependent measures. The data described as "Experiment 1 (fast)" included only the 8 fastest participants in Experiment 1

\begin{tabular}{|c|c|c|c|c|c|c|c|}
\hline \multirow[b]{2}{*}{ Experiment } & \multirow[b]{2}{*}{ Effect } & \multicolumn{3}{|c|}{ Mean Correct RT } & \multicolumn{3}{|c|}{ Mean Error Percentage } \\
\hline & & $F$ & $p$ & $\eta_{\mathrm{p}}^{2}$ & $F$ & $p$ & $\eta_{\mathrm{p}}{ }^{2}$ \\
\hline \multirow[t]{3}{*}{ 1a } & set size & 0.15 & .71 & .02 & 0.09 & .77 & .01 \\
\hline & distractor & 0.21 & .66 & .03 & 0.17 & .69 & .02 \\
\hline & IA & 0.03 & .87 & $<.01$ & 2.85 & .14 & .29 \\
\hline \multirow[t]{3}{*}{$1 b$} & set size & 0.11 & .75 & .02 & 0.02 & .89 & $<.01$ \\
\hline & distractor & 0.02 & .91 & $<.01$ & 14.98 & $<.01$ & .68 \\
\hline & IA & 0.05 & .83 & $<.01$ & 1.30 & .29 & .16 \\
\hline \multirow[t]{3}{*}{$1 \mathrm{c}$} & set size & 1.02 & .35 & .13 & 0.14 & .72 & .02 \\
\hline & distractor & 0.10 & .77 & .01 & 0.31 & .60 & .04 \\
\hline & IA & 0.05 & .83 & $<.01$ & 0.31 & .59 & .04 \\
\hline \multirow[t]{3}{*}{$1 \mathrm{~d}$} & set size & 0.38 & .55 & .04 & 0.39 & .55 & .04 \\
\hline & distractor & 0.27 & .62 & .03 & 1.79 & .21 & .17 \\
\hline & IA & 0.06 & .82 & $<.01$ & 1.79 & .21 & .17 \\
\hline \multirow[t]{3}{*}{1 (fast) } & set size & 0.04 & .85 & .01 & 0.13 & .73 & .02 \\
\hline & distractor & 0.55 & .48 & .07 & 0.92 & .37 & .12 \\
\hline & IA & 1.06 & .33 & .13 & 1.22 & .31 & .15 \\
\hline \multirow[t]{3}{*}{$2 \mathrm{a}$} & set size & 0.02 & .91 & $<.01$ & 0.22 & .65 & .03 \\
\hline & distractor & 0.01 & .95 & $<.01$ & 0.38 & .56 & .05 \\
\hline & IA & 2.36 & .17 & .25 & 1.10 & .33 & .14 \\
\hline \multirow[t]{3}{*}{$2 b$} & set size & 2.66 & .15 & .28 & 0.09 & .78 & .01 \\
\hline & distractor & 0.10 & .76 & .02 & 1.51 & .26 & .18 \\
\hline & IA & 8.06 & .03 & .54 & 0.09 & .78 & .01 \\
\hline \multirow[t]{3}{*}{3} & set size & 1.02 & .35 & .13 & 1.23 & .30 & .15 \\
\hline & distractor & 13.90 & $<.01$ & .67 & 0.18 & .69 & .03 \\
\hline & IA & 1.10 & .33 & .14 & 18.02 & $<.01$ & .72 \\
\hline \multirow[t]{3}{*}{4} & set size & 3.32 & .10 & .23 & 1.94 & .19 & .15 \\
\hline & distractor & 0.47 & .51 & .04 & 2.18 & .17 & .17 \\
\hline & IA & 0.02 & .89 & $<.01$ & 0.01 & .91 & $<.01$ \\
\hline
\end{tabular}

Fig. 2 Mean correct response times (RTs) in milliseconds from Experiments 1 and 3 as a function of set size and distractor condition
$\mathrm{A}=$ set size $\times$ distractor interaction; the $F$-tests had 1 and 7 $d f_{\mathrm{s}}$ in all experiments except for Experiment 1d, where $d f$ s were 1 and 9, and Experiment 4, where $d f$ s were 1 and 11 .

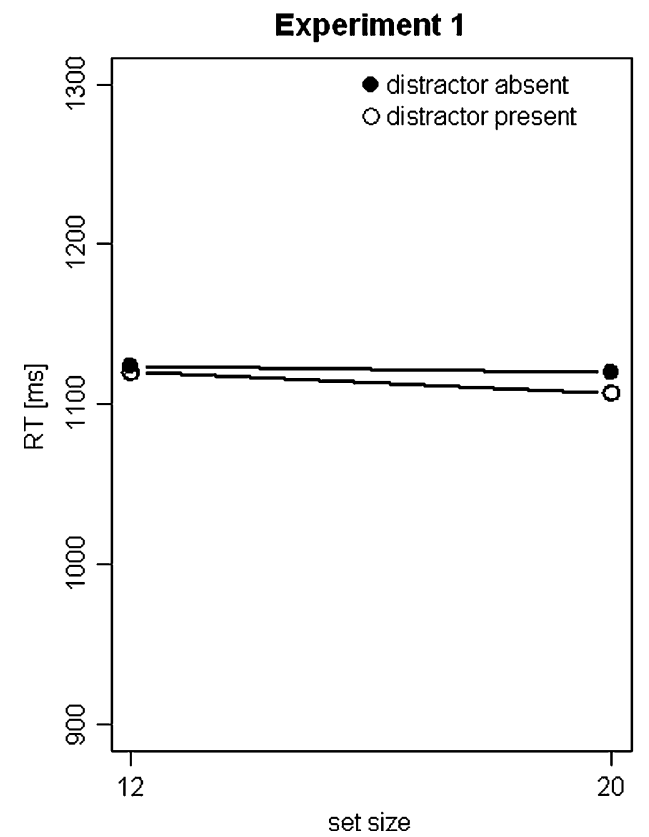

Experiment 3

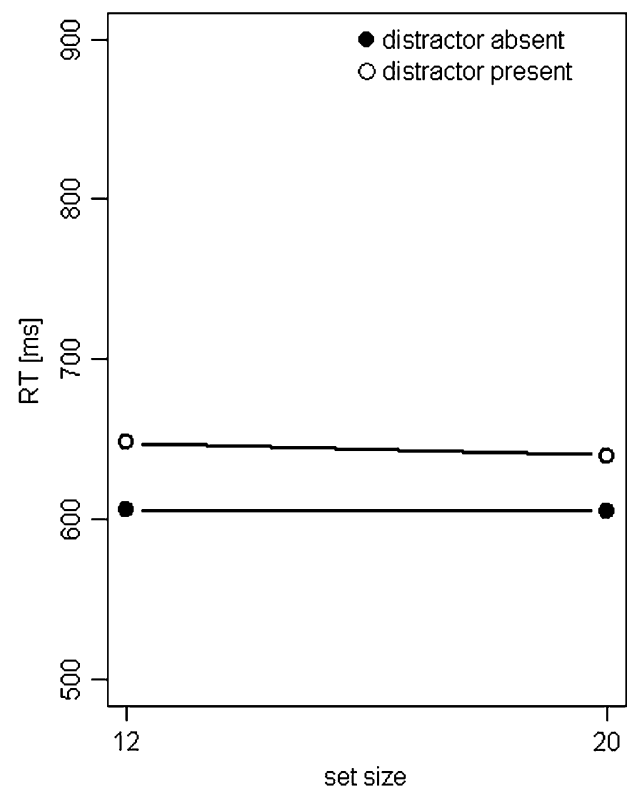


Fig. 3 Percentiles (5\%-95\%) of distractor present versus distractor absent response times (RTs) in milliseconds from Experiments 1 (left panel) and 4 (right panel)

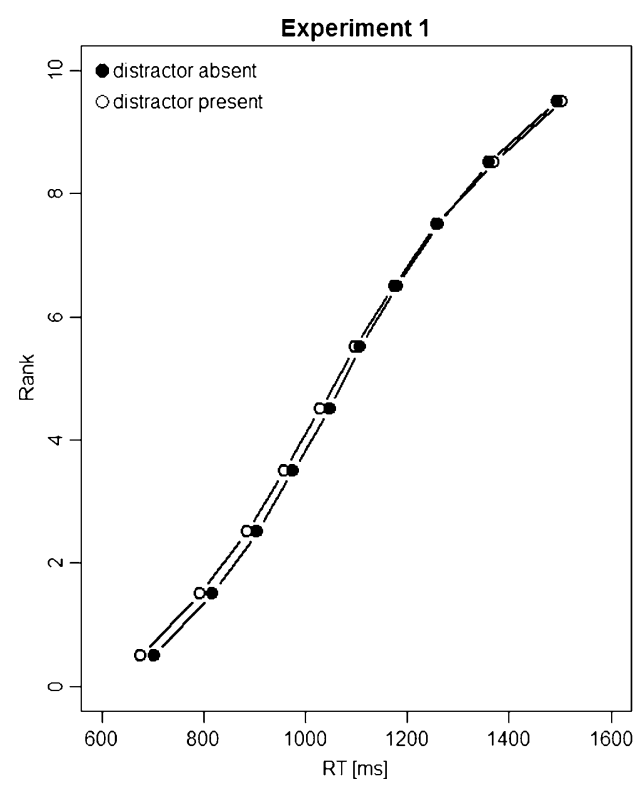

tion for error rates might indicate a possible distractor effect that occurred in the set size 12 condition. Note that this runs counter to Theeuwes' (2004) proposal, since a distractor effect should be more likely to emerge for large set sizes (i.e., 20) than for the smaller set size of 12. Second, although our experiments were a close replication, RTs were about 150 $200 \mathrm{~ms}$ longer in our Experiments 1a-1d, as compared with Experiment 1 in Theeuwes (2004). It is possible that slow responses mask attentional capture effects, which may be observable only with faster responses (see Theeuwes, 2010; Van der Stigchel, Belopolsky, Peters, Wijnen, Meeter, \& Theeuwes, 2009). Therefore, we additionally analyzed cumulative distribution functions and calculated percentiles $(5 \%, 15 \%, 25 \%, 35 \%, 45 \%, 55 \%, 65 \%, 75 \%, 85 \%$, and $95 \%)$ for each participant and distractor (absent vs. present) condition (see Fig. 3, left panel). The percentile $\times$ distractor interaction was not significant, $F(9,297)=1.16, p=.33, \eta_{\mathrm{p}}{ }^{2}=.03$. Furthermore, we tested for a distractor effect at each percentile separately, but no $t$-test revealed a significant effect, all $|t| \mathrm{s}(33) \leq 1.31, p \mathrm{~s} \geq .20$. In addition, we repeated the main analysis including only the data from the 8 fastest participants (see Tables 1 and 2 for detailed results). Even though the mean RTs were now almost in the range of those in Experiment 1 in Theeuwes (2004), no distractor effect was observed.

To summarize, we were not successful in four similar experiments to replicate Experiment 1 in Theeuwes (2004). In Experiments $2 \mathrm{a}$ and $2 \mathrm{~b}$, we introduced minor modifications and repeated our attempt at replication.

\section{Experiments 2a and 2b}

In Experiments 1a-1d, we failed to replicate the distractor effect observed in Experiment 1 in Theeuwes (2004).
Which factors could be responsible for these divergent results? One such factor might be the order of practice and test blocks, which is not explicitly mentioned in Theeuwes (2004). We therefore changed the order of practice and test blocks in Experiment 2a, to assess whether this factor may account for the different results of Theeuwes' (2004) study and our Experiment 1. A second explanation for our failure to replicate could be that the color singleton was simply not sufficiently salient to capture attention, although always falling inside the attentional window. This issue was addressed in Experiment $2 b$, where we made the distractor more salient by matching the colors' luminance.

\section{Method}

In general, these experiments were almost identical to Experiment 1, with two exceptions. In Experiment 2a, we changed the order of blocks: In contrast to the other experiments reported here, the participants first completed the practice trials in both distractor conditions, which were then followed by the test trials in both conditions (order of distractor conditions was counterbalanced across participants). In Experiment 2b, we lowered the brightness of all green stimuli to make the target and distractor isoluminant $\left(19.0 \mathrm{~cd} / \mathrm{m}^{2}\right)$. This manipulation should make the color distractor more salient, thereby facilitating attentional capture.

\section{Results and discussion}

Average mean correct RTs and mean error percentages for Experiments $2 \mathrm{a}$ and $2 \mathrm{~b}$ are summarized in Table 1, with the detailed ANOVA statistics given in Table 2. As in 
Experiments 1a-1d, neither set size nor distractor had significant main effects on RTs (only the interaction was significant in Experiment 2b). In other words, participants searched the display in parallel, but again no attentional capture was observed, despite the two introduced modifications. We conclude that neither the block order nor the perceived salience of the color distractor used in Experiments $1 \mathrm{a}-1 \mathrm{~d}$ was the reason for our failure to replicate Experiment 1 in Theeuwes (2004). Admittedly, we cannot exclude the possibility that a further increase of salience might induce a distractor effect, but this reservation will be addressed in Experiment 3.

\section{Experiment 3}

None of our (six) previous attempts to replicate Theeuwes's (2004) Experiment 1 were successful. Yet this experiment is crucial for the attentional window hypothesis, and our results, in combination with those of Leber and Egeth (2006), render this important empirical support less convincing. In fact, our results so far are well in line with a feature search mode (Bacon \& Egeth, 1994): Attention is set to a specific feature (in the present study, the diamond shape), and only stimuli that share this feature can capture attention involuntarily. Alternatively, however, it is still possible that our distractor (in Experiments 1 and 2) was, for some reason, simply not sufficiently salient to yield attentional capture. Therefore, we modified our design in a way that the target (diamond shape) was the only non-circle item-thus, a form singleton (Bacon \& Egeth, 1994, Experiment 1; Theeuwes, 1992). This would allow participants to use what Bacon and Egeth have termed the singleton search mode, and we consequently expected a parallel search plus a distractor effect now. Such a finding would weaken the remaining reservations related to our Experiments 1 and 2-namely, that the color distractor was not sufficiently salient to capture attention.

\section{Method}

This experiment was almost identical to Experiment 1, with one exception: In Experiment 3, the target (diamond shape) was the only non-circle item-thus, a form singleton (Bacon \& Egeth, 1994, Experiment 1; Theeuwes, 1992).

Results and discussion

Average mean correct RTs and mean error percentages for Experiment 3 are summarized in Table 1, with the detailed ANOVA statistics given in Table 2, and are presented visually in Figure 2 (right panel). Most notably, there now was a significant main effect of the distractor; thus, the distractor did capture attention when the observers could employ a singleton search mode. Importantly, this finding rules out the possibility that the distractor used so far was not sufficiently salient to capture attention.

\section{Experiment 4}

Our Experiments 1 and 2 were designed as close replications of Experiment 1 in Theeuwes (2004), but we never observed a distractor effect in parallel search (despite isoluminant targets and distractors in Experiment $2 \mathrm{~b}$ and $\mathrm{a}$ clear distractor effect in Experiment 3, where the target was a shape singleton). What procedural differences may still exist and be the cause for the discrepancy? In the Theeuwes (1992) study, the red and green luminance values were much lower than ours were, the background was dark gray (as compared with black in our study), and the color of the small line segments was white (as compared with green in our study). ${ }^{1}$ If we assume that these details apply to the 2004 study as well, they could account for the different results. In particular, these specifics of our experiments may also have slowed down responses, which may have blurred attentional capture effects (Theeuwes, 2010; Van der Stigchel et al., 2009). In Experiment 4, we therefore adopted these additional procedural details from the Theeuwes (1992) study.

\section{Method}

This experiment was similar to Experiments 1a-1d, with a few changes to incorporate procedural details from Theeuwes (1992). First, the luminance of the red and green stimuli was lowered to $5.5 \mathrm{~cd} / \mathrm{m}^{2}$, and the background color was set to dark gray $\left(0.4 \mathrm{~cd} / \mathrm{m}^{2}\right)$. Second, the color of the line segments was changed from green to white $\left(105 \mathrm{~cd} / \mathrm{m}^{2}\right)$.

\section{Results and discussion}

Average mean correct RTs and mean error percentages for Experiment 4 are summarized in Table 1, with the detailed ANOVA statistics given in Table 2. Results suggest a parallel search pattern but, despite the procedural changes, exhibit no effect of the distractor. In addition, we performed an analysis of the cumulative distribution functions as we did for Experiment 1 (see Fig. 3, right panel). The percentile $\times$ distractor interaction was not significant, $F(9$, 99) $=0.70, p=.56, \eta_{\mathrm{p}}{ }^{2}=.06$. Separate $t$-tests at each percentile were not significant as well, all $|t| \mathrm{s}(11) \leq 1.68, \mathrm{ps}$ $\geq .12$. In sum, the emerging picture is very similar to what we observed in Experiments 1 and 2.

\footnotetext{
${ }^{1}$ We thank Jan Theeuwes for directing our attention to these differences.
} 


\section{General discussion}

In the following section, we first summarize the results obtained from our experiments. We then consider possible further differences between our experiments and those in Theeuwes (2004) before discussing the implications for the attentional window hypothesis.

Parallel search without interference from a distractor

The attentional window hypothesis (Theeuwes, 2004) potentially provides a parsimonious explanation for attentional capture in visual search as an alternative to the different search modes favored by Bacon and Egeth (1994; see also Lamy \& Egeth, 2003): Observers do not engage in a specific search mode, but instead, they only set the attentional window to different sizes. If the search task is easy (e.g., when the target's salience is high), one may adopt a large attentional window yielding a parallel search. However, the more difficult the search task, the more likely one is to reduce the size of this window, resulting in a serial search. Assuming that sufficiently salient items inside this window interfere with the search for the target, parallel search should always yield attentional capture in the presence of such a (non-target) item (see also Leber \& Egeth, 2006). Experiment 1 in Theeuwes (2004) demonstrated exactly this in a paradigm that has previously been used to encourage the use of the feature search mode (Bacon \& Egeth, 1994), which, in turn, should prevent attentional capture-at least from static singletons (Lamy \& Egeth, 2003). In our Experiments 1, 2, and 4, we were not successful in replicating this crucial result of Theeuwes (2004): We did find parallel search, but we never found interference from a distracting color singleton. Thus, it seems that even (sufficiently) salient singletons inside the attentional window do not capture attention in an automatic and unavoidable way. In contrast, in our Experiment 3, the target was a form singleton, and here we found the expected interference from the distractor. This finding suggests that our failure to replicate cannot be accounted for by claiming that our distractor was simply not sufficiently salient.

\section{Differences between our study and Theeuwes (2004)}

First, the results reported here clearly differ from those of Experiment 1 in Theeuwes (2004) in that we never found a distractor effect. This is noteworthy since we tried to replicate the original experiment as closely as possible (Experiments 1 and 2) and even incorporated further potentially critical differences into Experiment 4. Second, an additional and obvious difference in the results relates to the overall RTs: Clearly, in our Experiments 1, 2, and 4, participants responded more slowly than in the Theeuwes
(2004) study. ${ }^{2}$ It has repeatedly been argued that bottom-up driven attentional capture may be blurred in the case of slow responses (e.g., Theeuwes, 2010; Van der Stigchel et al., 2009). In fact, a recent formulation suggests that the unavoidable attentional capture by bottom-up factors such as salience emerges only during a first wave of processing (Theeuwes, 2010; Van der Stigchel et al., 2009; see also Van Zoest, Donk \& Theeuwes, 2004). Later, however, top-down control gains more and more influence over selection (but see Ansorge, Kiss, Worschech, \& Eimer (2011) for evidence that even in the first stage, top-down settings play an important role). Admittedly, we cannot exclude the reemergence of a distractor effect with shorter RTs than in the present experiments. It is important to remember, though, that additional analyses (cumulative distribution function, extreme group analysis) of the data from Experiments 1 and 4 exhibited no sign of such an effect, even for the shortest RTs. Why were our participants slower than those in the Theeuwes (2004) study? First, the differences may be due to unspecific experimental or laboratory settings (different response keys, different computers, and so forth). Yet given that we collected the data at three different German universities, all yielding comparable results, we suspect that such an explanation is unlikely. In addition, we conducted an experiment attempting to replicate Theeuwes' (2004) Experiment 2. Here, set sizes of 5 and 9 resulted in a serial search without a distractor effect-exactly replicating the original study. ${ }^{3}$ Second, although speculative, we cannot exclude subtle differences in the populations from which the samples were drawn. Only a few of our participants had prior experience with visual search experiments such as those employed in the present study. It might be that the participants in the Theeuwes (2004) study were more experienced in this domain, thus yielding shorter RTs overall. If this is true, we cannot exclude certainly the occurrence of distractor effects after more intense training.

\section{Theoretical implications}

Leber and Egeth (2006) showed that even when the target is a singleton, it is possible to observe parallel search without attentional capture from a sufficiently salient distractor, when participants are previously trained in a search task requiring the feature search mode. Our research extends this

\footnotetext{
2 The fact that in many other studies using this (or a similar) paradigm, RTs were shorter than in our Experiments 1, 2, and 4 may call into question the validity of our results. However, we find this premature, since (if set size was varied at all) the number of items typically varied between 3 and 9 except for Experiment 1 in Theeuwes (2004). Thus, it is far from being clear how set sizes of 12 and 20 (where items are even arranged in different ways) affect RTs in this specific paradigm and display setup.

${ }^{3}$ More precisely, we observed a set size effect, indicating a (partly) serial search, $F(1,7)=22.54, p<.01, \eta_{\mathrm{p}}{ }^{2}=.76$. The distractor had no significant effect, $F(1,7)<0.01, p=.99, \eta_{\mathrm{p}}{ }^{2}<.01$. The interaction was not significant either, $F(1,7)=0.12, p=.74, \eta_{\mathrm{p}}{ }^{2}=.02$.
} 
finding by showing that even without specific training, it is possible to observe parallel search without attentional capture, and this violates a key assumption of the attentional window hypothesis (Theeuwes, 2004). In fact, such a pattern may even be the case in the study by Belopolsky and Theeuwes (2010). In their Experiment 2, no distractor effect was observed in their focused condition - presumably, showing signs of serial search. Note, however, that the reported search slope for the distractor absent condition was only $5.5 \mathrm{~ms} /$ item - a slope that arguably can also be interpreted as reflecting a parallel search (for the distractor present condition, the search slope was essentially zero).

We suggest that our results are better reconcilable with the notion of a feature versus a singleton search mode (Bacon \& Egeth, 1994): In Experiments 1, 2, and 4, participants were obliged to rely on the feature search mode and, thus, were not susceptible to attentional capture by the irrelevant color singleton. In contrast, in Experiment 3, the singleton search mode could have been exploited, and exactly as we found, participants were susceptible to interference from the very same color singleton. However, note that we successfully replicated Theeuwes's (2004) Experiment 2 (see Footnote 3). The finding from this experiment is different from Experiments 2 and 3 in Bacon and Egeth, who suggested that their data reveal a parallel search. Does this pose a problem for the search mode approach (Bacon \& Egeth, 1994)? We see two possible interpretations that might reconcile this finding with the assumption of a feature search mode (Bacon \& Egeth, 1994). First, one may assume that, in principle, the feature search mode can be applied independently of whether search is parallel or (partially) serial. In fact, the task in the Bacon and Egeth study may have been easier than that in our replication of Theeuwes' (2004) Experiment 2 and that in his original experiment, since in the latter studies, density was lower, resulting in less salient targets and distractors. If one accepts this, the critical result would be compatible with both the feature search mode (Bacon \& Egeth, 1994) and the attentional window hypothesis (Theeuwes, 2004), but given the serial search pattern, the data from this experiment simply cannot be used to distinguish between the two accounts. Second, an alternative would be to merge both ideas: The size of the attentional window is set in a top-down manner dependent on, for example, task difficulty. But regardless of its size, to at least some degree, some additional top-down settings determine which features are attended to and which can be ignored efficiently. In other words, under top-down control is not only the size of the attentional window, but also what happens to its content.

Some final comments

Overall, our data appear more consistent with the search mode approach (Bacon \& Egeth, 1994) than with the attentional window hypothesis (Theeuwes, 2004). However, we wish to emphasize that it is clear that our data do not resolve the long-debated controversy about the (relative) contributions of bottom-up and top-down mechanisms to visual selection. Still, we believe that our data put further constraints on current theorizing. But, not surprisingly, more research needs to be carried out. In particular, efforts should be made to thoroughly identify the factors that may have caused the RT differences in our study and the study by Theeuwes (2004).

Acknowledgements We gratefully thank Gisela Müller-Plath for her important suggestions and support in the course of this research. Furthermore, we thank Gisela Müller-Plath, Jan Theeuwes, Gernot Horstmann, Andrew Leber, and Jeremy Wolfe for their valuable comments and suggestions on a previous version of the manuscript.

\section{References}

Ansorge, U., Kiss, M., Worschech, F., \& Eimer, M. (2011). The initial stage of visual selection is controlled by top-down task set: New ERP evidence. Attention, Perception, \& Psychophysics, 73, 113122.

Bacon, W. F., \& Egeth, H. E. (1994). Overriding stimulus-driven attentional capture. Perception \& Psychophysics, 55, 485-496.

Belopolsky, A. V., \& Theeuwes, J. (2010). No capture outside the attentional window. Vision Research, 50, 2543-2550.

Belopolsky, A. V., Zwaan, L., Theeuwes, J., \& Kramer, A. F. (2007). The size of an attentional window modulates attentional capture by color singletons. Psychonomic Bulletin \& Review, 14, 934 938.

Folk, C. L., Remington, R. W., \& Johnston, J. C. (1992). Involuntary covert orienting is contingent on attentional control setting. Journal of Experimental Psychology: Human Perception and Performance, 18, 1030-1044.

Lamy, D., \& Egeth, H. E. (2003). Attentional capture in singletondetection and feature-search modes. Journal of Experimental Psychology: Human Perception and Performance, 29, 10031020.

Leber, A. B., \& Egeth, H. E. (2006). It's under control: Top-down search strategies can override attentional capture. Psychonomic Bulletin \& Review, 13, 132-138.

Pashler, H. (1988). Familiarity and visual change detection. Perception \& Psychophysics, 44, 369-378.

Theeuwes, J. (1991). Cross dimensional perceptual selectivity. Perception \& Psychophysics, 50, 184-193.

Theeuwes, J. (1992). Perceptual selectivity for color and shape. Perception \& Psychophysics, 51, 599-606.

Theeuwes, J. (1994). Endogenous and exogenous control of visual selection. Perception, 23, 429-440.

Theeuwes, J. (2004). Top-down search strategies cannot override attentional capture. Psychonomic Bulletin \& Review, 11, 65-70.

Theeuwes, J. (2010). Top-down and bottom-up control of visual selection. Acta Psychologica, 135, 77-99.

Van der Stigchel, S., Belopolsky, A. V., Peters, J. C., Wijnen, J. G., Meeter, M., \& Theeuwes, J. (2009). The limits of top-down control of visual attention. Acta Psychologica, 132, 201-212.

Van Zoest, W., Donk, M., \& Theeuwes, J. (2004). The role of stimulusdriven and goal-driven control in saccadic visual selection. Journal of Experimental Psychology: Human Perception and Performance, 30, 746-759. 